
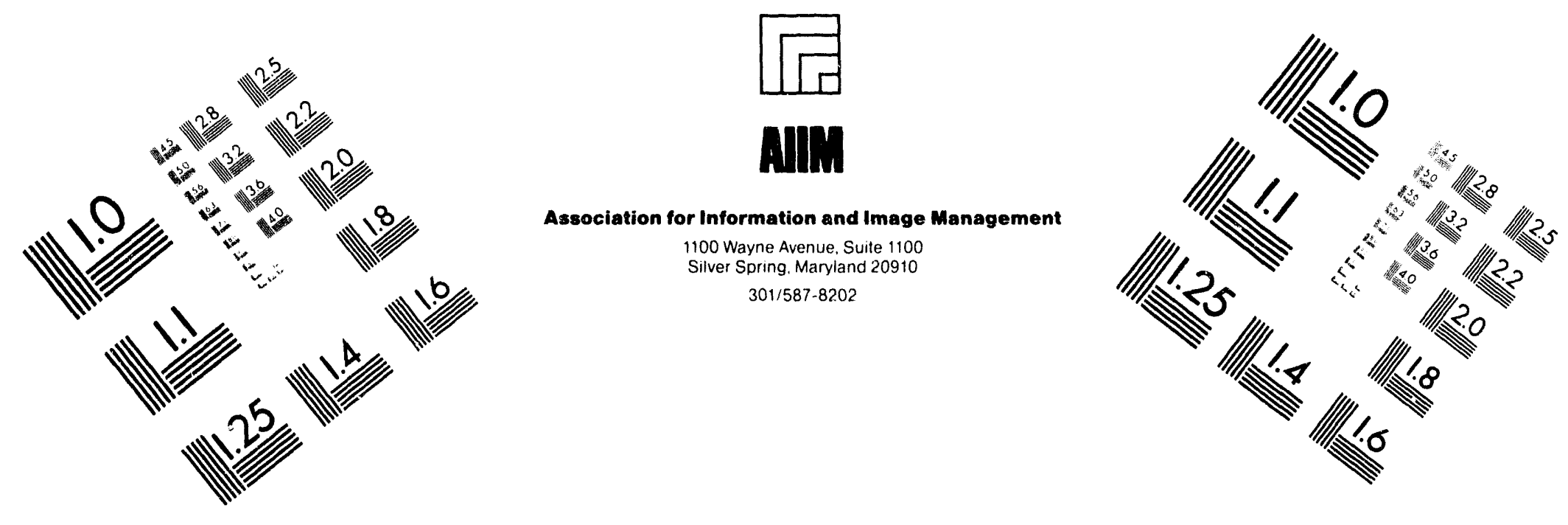

\title{
Centimeter
}

$\begin{array}{llllllllllllllll}1 & 2 & 3 & 4 & 5 & 6 & 7 & 8 & 9 & 10 & 11 & 12 & 13 & 14 & 15 & \mathrm{~mm}\end{array}$

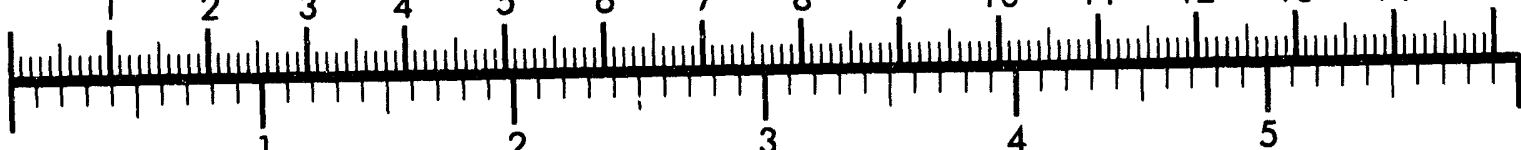
Inches
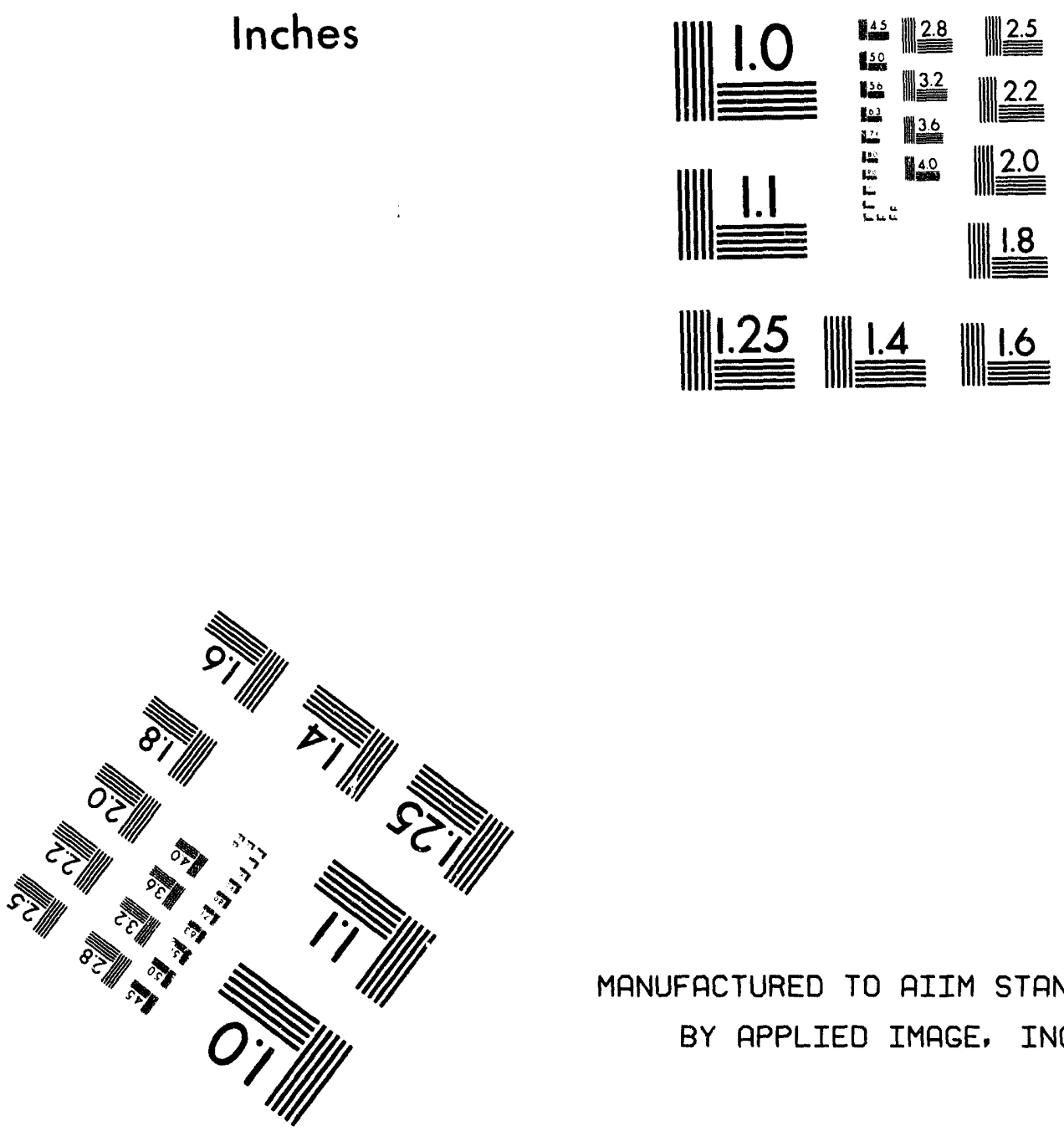

MANUFACTURED TO AIIM STANDARDS

BY APPLIED IMAGE, INC.

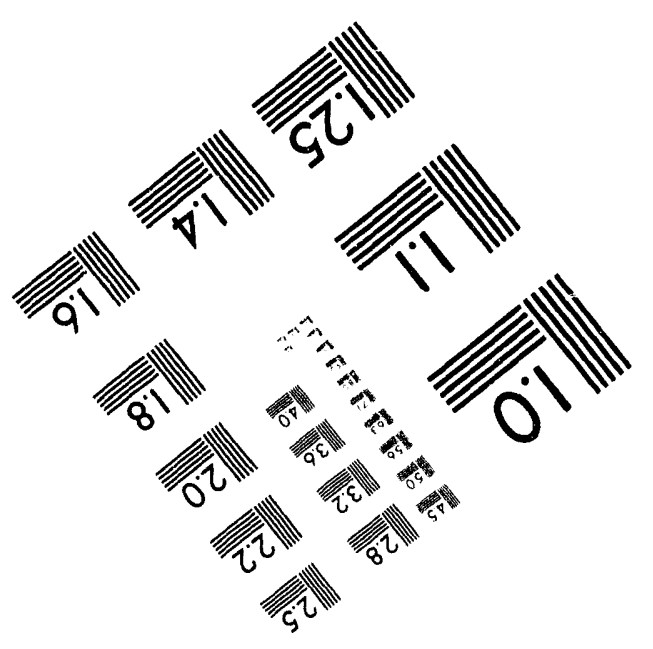



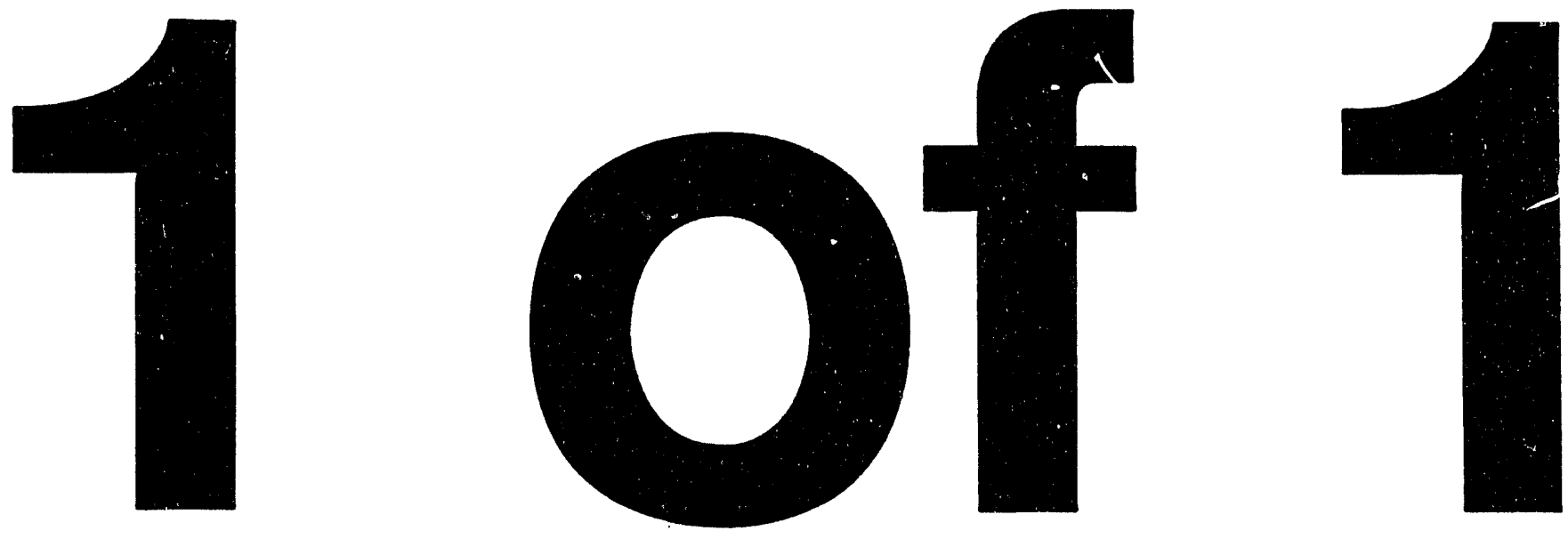


\title{
CUNF-940586--1
}

\author{
Proceedings \\ Engineering Foundation Conference on \\ Materials Processing and Advanced Application of Lasers \\ Palm Coast, Florida \\ May 1-6, 1994
}

\section{COMPUTATIONAL MODELING OF PHYSICAL PROCESSES DURING LASER ABLATION}

\author{
C. L. Liu, J. N. Leboeuf, R. F. Wood, D. B. Geohegan
}

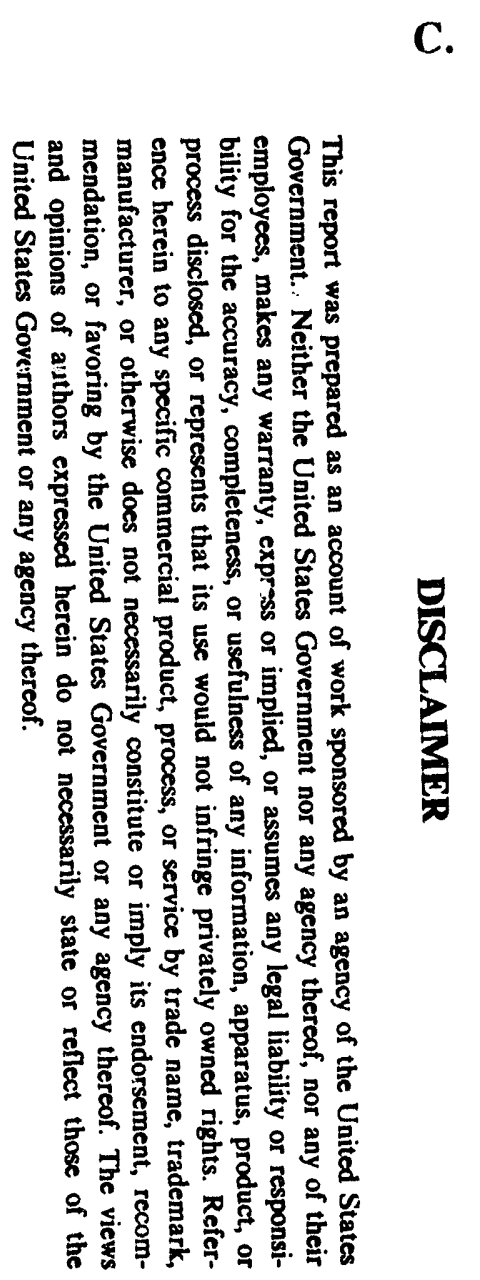

The submilted manuscript has been authored by contractor of the U.S. Govemment under Accordingly, the U.S. Govemment retains Acciexclusive myalty-free license to publish or

reproduce the published form of thi

contribution, or lllow others to do so, for U.S.

Govemment purposes."

J. M. Donato, K. R. Chen, and A. A. Puretzky

$\frac{8}{3}$

\author{
SOLID STATE DIVISION \\ OAK RIDGE NATIONAL LABORATORY \\ Managed by \\ MARTIN MARIETTA ENERGY SYSTEMS, INC. \\ Under \\ Contract No. DE-AC05-84OR21400 \\ With the \\ U. S. DEPARTMENT OF ENERGY \\ OAK RIDGE, TENNESSEE
}

June 1994

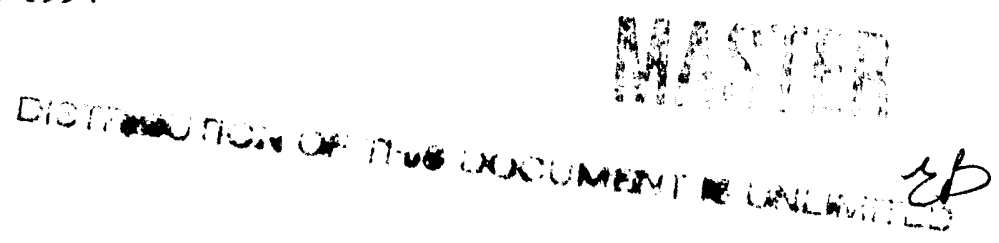




\title{
COMPUTATIONAL MODELING OF PHYSICAL PROCESSES DURING LASER ABLATION
}

\section{L. Liu, J. N. Leboeuf,* R. F. Wood, D. B. Geohegan, J. M. Donato, ${ }^{* *}$ K. R. Chen, ${ }^{*}$ and A. A. Puretzky Solid State Division, Oak Ridge National Laboratory Oak Ridge, TN 37831-6032}

\begin{abstract}
A combined theoretical and experimental effort to model various physical processes during laser ablation of solids using a variety of computational techniques is described. Currently the focus of the modeling is on the following areas: (a) rapid transformations through the liquid and vapor phases under possibly nonequilibrium thermodynamic conditions induced by laser-solid interactions, (b) breakdown of the vapor into a plasma in the early stages of ablation through both electronic and photoionization processes, (c) hydrodynamic behavior of the vapor/plasma during and after ablation, and (d) the effects of initial conditions in the vapor, in particular, the nature of the initial velocity distribution, on the characteristics of subsequent vapor expansion. The results from the modeling will be compared with experimental observations where possible.

\footnotetext{
*Fusion Energy Division, ORNL

**Engineering Physics and Mathematics Division, ORNL
}

Key words: computational modeling, laser ablation, laser annealing, vapor breakdown, particle hydrodynamic, gas dynamic. 


\section{INTRODUCTION}

Materials processing with lasers is considered by many to be one of the techniques which can have great impact on materials science and engineering in the 90 's and beyond. ${ }^{1}$ One of the main techniques for laser materials processing is Pulsed Laser Deposition (PLD) for thin film growth. The advantages of PLD compared to other techniques include, novel epitaxial and low temperature growth of homogeneous and heterogeneous films by utilizing energetic species, stoichiometric ablation of constituent species of the target, and growth of metastable phases layer-by-layer under nonequilibrium ablation conditions. However, there are still some issues, such as inclusion of particulates in the growing films and spatial non-uniformities of the films over large areas, that must be resolved before PLD can be widely used for industrial applications. While experimentalists are trying to find optimal approaches for thin film growth, a systematic effort in modeling of various physical processes during PLD is needed. In this paper, we present preliminary results from theoretical modeling of laser-ablation phenomena in silicon using a variety of computational techniques. One faces the challenge not only to better understand the fundamentals of the processes, but also to utilize various computational techniques to introduce the governing physics into the modeling. Consequently, we have chosen to work with silicon because it is an elemental material and because of the vast amount of results now available from laser annealing studies. Also, there is a strong experimental program on laser ablation at our laboratory and this allows close coordination between the theoretical and experimental research.

Figure 1 shows a schematic of laser ablation for which one can categorize the following

processes: (i) laser-solid target interactions, (ii) laser-plume interactions, (iii) plasma expansion and transport toward the substrate, (iv) plasma-substrate interactions.

As for laser-solid interactions, we primarily consider extensions of the laser annealing process that has been extensively investigated over the last $10-15$ years. ${ }^{2}$ These extensions 
enable the modeling to deal with pulse energy densities that result in extensive vaporization of the near-surface region of the target. Upon evaporation, the laser radiation interacts simultaneously with the target and the plume, partitioning the laser energy among them. To illustrate this phase of the laser ablation process we have chosen to review work on laser irradiation of amorphous $\mathrm{Si}$ (a-Si) because it demonstrates the wide range of capabilities of the LASER8 computer programs ${ }^{3}$ on which this part of the research is based.

In the plume, many processes such as those of thermal, photoelectric, hydrodynamic, electronic, and electromagnetic origins may occur simultaneously. One of the results of laser-plume interactions is vapor breakdown, i.e., plasma ignition, during which electron and ion densities increase rapidly and at the same time the neutral density in the plume decrease dramatically. Vapor breakdown can be defined as complete ionization of the vapor. ${ }^{4}$ It is crucial to understand the breakdown phenomenon since it usually sets up the final state of the plume, which has a dominant effect on the energetics of the particles in the plume and therefore on the growth of thin films.

Once a plasma is generated it will expand during transport towards the substrate either freely or adiabatically in vacuum, or through frontal shock wave propagation in the ambient gas, or in mixed fashion in the case of low pressure of the background gas. During such a process, the plasma exhibits hydrodynamic behavior while thermalization occurs within the plasma. Distributions of various quantities inside the plasma at the initial stage of laser ablation can have a dramatic effect on the characteristics of subsequent expansion and transport of the plasma and thus they play a critical role for thin-film deposition on the substrate.

Finally, when the plasma reaches the substrate, energetic particles are deposited and thin films are grown. The sticking coefficient of the energetic particles may not be close to unity and some particles can be reflected back, thus creating shock waves within the depositing plasma. The effects of the particle energetics on the quality of thin films are extremely important in understanding PLD processes. 
In the following sections, we will briefly describe each of the four areas we have been working on in the course of theoretical modeling of laser-induced processes, namely, (a) laser melting and vaporization of the target, (b) vapor breakdown, (c) 1-D gas dynamic modeling of initial plasma expansion and transport, (d) a 2-D particle hydrodynamic model for plasma expansion and transport.

\section{PULSED LASER MELTING AND EVAPORATION}

In order to model the effects of laser radiation on solids, one may have to deal with a variety of phenomena and issues, such as melting, solidification, overheating and undercooling, nucleation, coexisting multiple phases anc rapidly moving multiple phase boundaries, interfacial kinetics, temperature- and phase-dependent optical and thermal properties of the materials, etc. In the LASER8 programs, ${ }^{5}$ finite difference (FD) methods were used to solve heat flow equations in terms of enthalpy and temperature, with the former being used to determine phase changes and the latter to determine heat flow. A concept of state arrays was developed to monitor phase status and other parameters in each finite difference cell. These models allow nonequilibrium melting and solidification to occur at temperatures other than the thermodynamic phase transformation temperatures. Therefore, interfacial undercooling and overheating can be included in the modeling and many types of nucleation events can be simulated through the state array feature. These capabilities may become quite useful in studying laser ablation.

Applications of the models to laser annealing of silicon have shown a variety of behavior, including so-called 'explosive crystallization' during nucleation in undercooled liquid Si prepared by pulsed-laser melting of a-Si, which we will use here for illustrative purposes. Figure 2 shows a graphic output of the time evolution of the phases in each finite difference cell for laser annealing of a $195 \mathrm{~nm}$ a-Si layer on a crystal Si substrate. Each letter represents a FD cell of 100 angstroms in thickness (the letters' correspondence: Aamorphous, C-crystal, M-mixed phase, S-undercooled liquid Si, L-liq.uid Si, F-fine-grained 
polycrystalline $\mathrm{Si}$, and P-large-grained polycrystalline $\mathrm{Si}$ ). In the left panel with encrgy fluence of $0.15 \mathrm{~J} / \mathrm{cm}^{2}$, we see that at $15 \mathrm{nsec}$ in the laser pulse, the surface begin to melt and at $37 \mathrm{nsec}$, fine-grained polycrystalline Si begin to nucleate. Since nucleation of the $\mathrm{F}$ cells releases the latent heat into the surrounding liquid, the excess heat in the undercooled liquid $\mathrm{Si}$ will drive the liquid deep into the amorphous layer, causing the explosive crystallization. The middle and right panels show the behavior at higher laser energy fluences. At an energy fluence of $0.4 \mathrm{~J} / \mathrm{cm}^{2}$, a mixture of normal and explosive crystallization is seen, with simultaneous propagation of a buried liquid layer into the solid and a more normal return of the melt front to the surface.

The approach employed to simulate laser annealing has been extended to treat the initial stages of the laser-ablation process up through vaporization. In fact, similar techniques can be used to simulate certain aspects of the early states of plasma formation.

\section{VAPOR BREAKDOWN}

A simple kinetic model was developed to simulate the vapor breakdown during ablation of a Si target. Two absorption mechanisms are considered for vapor heating through laser beam-plume interaction, i.e., inverse bremsstrahlung (IB) and photoionization of excited states.

Two necessary conditions are required in order for the vapor to breakdown: "priming" electrons and an electron density acceleration mechanism. There are many possible sources for "priming" electrons, such as (1) thermal ionization as described by the Saha equation under conditions of local thermal equilibrium; (2) thermionic emission either from the ablated surface or from heated liquid droplets; (3) shock heating and ionization; (4) surface defects which are thermally decoupled from the bulk (and usually are reservoirs of negative charge); (5) avalanche generation of electrons in the solid near the surface. Here we simply take the electron density obtained from thermal ionization at the surface temperature as the "priming" electrons. 
As for electron density acceleration, electron-impact excitation and ionization as well as photoionization of excited states were considered. Figure 3 shows a schematic of the processes involved in the kinetic model. From ground to excited state (energy = $5.0 \mathrm{eV}$ ), electron-impact excitation was taken as a forward process, and electron-impact deexcitation and spontaneous deexcitation of excited states due to the lifetime of the excited states were taken as reverse processes. From the excited state to ionized state, electron-impact ionization and photoionization of the excited state were taken as forward processes, and three-body recombination was taken as reverse process.

The rates of various processes were calculated according to the formulas given by Zel'dovich and Raizer. ${ }^{6}$ The rate equations were then solved for five quantities, namely, densities of electrons, neutrals, and excited states, and the temperatures of electrons and heavy-body particles (ions, neutrals, and excited states), much as was done earlier by Rosen et al. ${ }^{7}$ Resupply of the vapor by evaporation and dilution of it due to expausion were also taken into account in the calculations. Only singly charged particles were considered and for simplicity, a square pulse was used in the calculations.

A typical output from the kinetic model for a laser energy fluence of $6.0 \mathrm{~J} / \mathrm{cm}^{2}$ is given in Figure 4. The densities of electrons and excited states increase sharply at $48.0 \mathrm{nsec}$, and at the same time, the density of neutrals decreases by several orders of magnitudes, indicating vapor breakdown. 'The breakdown occurred within $1.0 \mathrm{nsec}$. Similar calculations were also carried out for higher energy fluences, $20.0 \mathrm{~J} / \mathrm{cm}^{2}$ and $30 \mathrm{~J} / \mathrm{cm}^{2}$, in particular.

Total breakdown time, defined here as the sum of evaporation time and vapor breakdown time, was also obtained. The evaporation time was obtained from the 1-D thermal model discussed in section II. The total breakdown times at energy fluences of $6.0 \mathrm{~J} / \mathrm{cm}^{2}$ and $20.0 \mathrm{~J} / \mathrm{cm}^{2}$ were calculated to be $70.0 \mathrm{nsec}(22.0 \mathrm{nsec}$ for evaporation and $48.0 \mathrm{nsec}$ for vapor breakdown) and $24.0 \mathrm{nsec}(6.0 \mathrm{nsec}$ for evaporation and $18.0 \mathrm{nsec}$ for vapor breakdown), respectively. The results indicated that at energy fluence of $6.0 \mathrm{~J} / \mathrm{cm}^{2}$ the total breakdown surprisingly occurred toward the end of the laser pulse which had a 
FWHM of 46.0 nesc. In this case, one may not see the vapor breakdown visibly by means of light emission. On the other hand, at an energy fluence of $20.0 \mathrm{~J} / \mathrm{cm}^{2}$, one may rcadily see the breakdown in the time frame $(24.0 \mathrm{nsec})$ of laser pulse 'uration since the total breakdown time calculated is much less than the FWHM pulse duration (46.0 nsec). This is qualitatively consistent with our experimental observations of $\mathrm{Si}$ target ablation using a $\mathrm{KrF}$ laser.

\section{GAS DYNAMIC MODELING OF PLASMA EXPANSION AND TRANSPORT}

One-dimensional coupled gas dynamic equations ${ }^{6}$ describing the conservation of mass, momentum, and energy were solved numerically to simulate plasma expansion and transport. This implementation is similar to that used by Vertes et al., ${ }^{8,9}$ except that the Rusanov scheme ${ }^{10}$ is used to advance the quantities in the hydrodynamic equations as a function of time. The Rusanov scheme was chosen since it is capable of describing shock waves approximately if there are any during plasma expansion and transport. The main equations used are:

Mass, momentum, and energy;

$$
\frac{\partial}{\partial t}\left\{\begin{array}{l}
\rho \\
\rho u \\
\rho\left(e+\frac{u^{2}}{2}\right)
\end{array}\right\}=-\frac{\partial}{\partial x}\left\{\begin{array}{l}
\rho u \\
\rho u^{2}+p \\
\rho u\left(e+\frac{p}{\rho}+\frac{u^{2}}{2}\right)
\end{array}\right\}+\left\{\begin{array}{l}
0 \\
0 \\
\alpha \Phi
\end{array}\right\}
$$

Equation of state;

$$
p=(1+\eta) \rho \frac{k_{B} T}{M}
$$

Internal energy density;

$$
\rho e=\frac{\rho}{M}\left[\frac{3}{2}(1+\eta) k_{B} T+\eta I_{p}\right]
$$

Saha equation for ionization;

$$
\frac{\eta^{2}}{1-\eta}=\frac{M}{\rho}\left(\frac{2 \pi m k_{B} T}{h^{2}}\right)^{3 / 2} e^{-I_{\mathrm{p}} / k_{B} t}
$$


where $\rho$ is the local particle density, $\mathrm{p}$ the local pressure, $\mathrm{u}$ the local fluid vclocity, $\rho \mathrm{e}$ the local internal energy density, $\Phi$ the average laser energy, $\alpha$ the linear absorption coefficient (which is taken as the sum of electron-neutral IB, electron-ion IB, and plasma resonance absorption coefficients), $\eta$ the ionization fraction, and $I_{p}$ the ionization energy of neutrals. The Newton-Raphson scheme was used to solve the equations of internal energy density and ionization simultaneously and thus obtain the temperature and ionization fraction.

Initial profiles of density, temperature, and velocity were obtained from our 1-D thermal model of section II and were used as input for the hydrodynamic modeling. A typical output for three of the variables, namely, local fluid velocity (top panel), local sound velocity (middle panel), and local ionization fraction (bottom panel), is given at three different times in Fig. 5. At the early time of laser irradiation (98.0 nsec pulse at energy fluence of $6.0 \mathrm{~J} / \mathrm{cm}^{2}$ ) when the plasma just begins to leave the target surface, a redeposition process occurs as indicated by the negative fluid velocity at the left frame of the top panel. This may be the cause of the small peak in the fluid velocity at a later time shown in the middle frame of the top panel. In addition, we also see that a shock front develops whenever the fluid velocity is larger than the local sound velocity, as shown in the middle frame of the top panel. After the laser irradiation was shut off, the plasma expands adiabatically. During adiabatic expansion, the thermal energy of the plasma can be transferred into kinetic energy of the particles and the increase in the fluid velocity can be seen in the right frame of the top panel. Shock heating and ionization were also indicated by the local ionization fraction in the right frame of the bottom panel.

\section{PARTICLE HYDRODYNAMIC MODELIIVG OF PLASMA EXPANSION AND TRANSPORT}

A 2-D particle hydrodynamic model has been developed by Leboeuf and collaborators $^{11,12}$ to trace the position and velocity of individual particles in a plasma. The main equations used are as follows: 
Particle equations of motion:

$$
\begin{gathered}
\frac{d \mathbf{x}_{j}}{d t}=\mathbf{v}_{j} \\
\frac{d \mathbf{v}_{j}}{d t}=-\frac{1}{\rho} \nabla P-\nu\left(\mathbf{v}_{j}-\mathbf{v}_{f}\right)
\end{gathered}
$$

Equation of state:

$$
P=\text { const. } X \rho \gamma
$$

Density and fluid velocity:

$$
\begin{gathered}
\rho=\sum_{j} S\left(\mathbf{x}-\mathbf{x}_{j}\right) \\
\mathbf{V}_{f}=\sum_{j} \mathbf{v}_{j} S\left(\mathbf{x}-\mathbf{x}_{j}\right) / \sum_{j} S\left(\mathbf{x}-\mathbf{x}_{j}\right)
\end{gathered}
$$

The subscript $j$ denotes the quantities for individual particles, the subscript $f$ for the fluid quantities, $\gamma$ the ratio of specific heat $\left(\mathrm{C}_{p} / \mathrm{Cv}\right), \mathrm{n}$ the viscous term, and $S$ denotes the shape function associated with each particle. The summation is for all the particles in each grid. Acceleration of particles is driven by the pressure gradient while a viscous force exerts a drag on the particles. Density and fluid velocities are accumulated on each grid point from particle positions and velocities using an area weighting method.

Plasma expansion and transport were simulated by setting up different initial velocity distributions for the particles. Figure 6 shows the characteristics of plasma expansion and transport with uniform initial velocity distribution in the $x$ direction. The plasma was initially confined within a pellet and then released into vacuum with a large fluid velocity. The plasma evolved into an elliptic ball shape sometime later and eventually hit the substrate where the boundary was set up to be a perfectly reflecting wall.

Background gas may play an important role during laser ablation. Simulations with and without background gas were also carried out for zero or small fluid velocities, i.e., free expansion of the plasma. Figure 7 shows the contour lines of particle density during plasma expansion into both vacuum and background gas. The plasma has expanded with time, but did not travel far from the target surface. The plasma is more confined in a 
background gas due to the collisions between particles in the plasma and particles in the gas. We also see that there is an area in which the contour line density seems to be high. This is further illustrated by 3-dimensional isosurface plots of particle density, as shown in Figure 8. The plasma is expanding from the right to the left and sometime later a small peak appears near the target surface, which is presumably due to the reflected particles. A peak is also developed in front of the plasma due to the interaction with the background gas. These peaks are highly suggestive of shock waves. If they correspond to local densities of excited states, one can probably see the light emitted from the locations of the peaks due to photon emission. ${ }^{13}$

\section{SUMMARY}

The results from preliminary theoretical modeling indicate the following conclusions:

- Laser annealing phenomena can be modeled by 1-D and 2-D thermal models with realistic treatments of nonequilibrium thermodynamic phase transformations. The thermal approach used can be applied to other systems and phenomena.

- Electronic and photoelectric processes may be necessary for modeling of vapor breakdown. Breakdown times predicted based on a simple kinetic model are qualitatively consistent with experimental observations.

- Modeling of vapor expansion and transport have revealed the following phenomena:

- Formation and propagation of shock waves

- Shock heating and ionization

- Dramatic effects of initial velocity distribution on the characteristics of subsequent plasma expansion and transport

- Confinement of plasma by a background gas 


\section{ACKNOWLEDGMENTS}

This research was sponsored by the Division of Materials Sciences, U.S. Department of Energy under Contract No. DE-AC05-84OR21400 with Martin Marietta Energy Systems, Inc., and in part by an appointment to the Oak Ridge National Laboratory Postdoctoral Research Associates Program administered jointly by the Oak Ridge National Laboratory and the Oak Ridge Institute for Science and Education. 


\section{REFERENCES}

1. See, for example, many of the papers in the Proceedings of this conference and other references therein.

2. Several general references on this subject are Pulsed Laser Processing of Semiconductors, Vol. 23 of the Semiconductors and Semimetals series, edited by R. F.

Wood, C. W. White, and R. T. Young (Academic, New York, 1984); Laser Annealing of Semiconductors, edited by J. M. Poate and J. W. Mayer (Academic, New York, 1982); and many volumes in the series of Proceedings of the Materials Research Society, published by North-Holland.

3. R. F. Wood and G. A. Geist, Phys. Rev. Lett. 57, 873 (1986); R. F. Wood and G. A. Geist, Phys. Rev. B 34, 2606 (1986).

4. G. M. Weyl, in: Laser-Induced Plasmas and Applications, ed. by Radziemski and Cremers, Marcel Dekker, inc., New York and Basel, 1986.

5. A $2 \mathrm{D}$ version of the LASER 8 program was developed several years ago; it will be described in a forthcoming publication.

6. Ya. B. Zel'dovich and Yu. P. Raizer, Physics of Shock Waves and High-Temperature Hydrodynamic Phenomena, Academic Press, New York and London, 1966.

7. D. I. Rosen, J. Mitteldorf, G. Kothandaraman, A. N. Pirri, and E. R. Pugh, J. Appl. Phys. 53, 3190 (1982).

8. A. Vertes, P. Juhasz, M. D. Wolf, and R. Gijbels, Scanning Microscopy 2, 1853, 1988.

9. L. Balazs, R. Gijbels, and A. Vertes, Analytical Chemistry 63, 3141991.

10. V. V. Rusanov, J. Comp. Math. Math. Phys. USSR, No. 2 (1962); G. A. Sod, J. Comput. Phys., 1 (1978).

11. J. N. Leboeuf, T. Tajima, and J. M. Dawson, J. Comput. Phys. 31, 379 (1979).

12. F. Brunel, J. N. Leboeuf, T. Tajima, J. M. Dawson, M. Makino, and T. Kamimura, J. Comput. Phys. 43, 268 (1981).

13. D. B. Geohegan, Appl. Phys. Lett. 60, 2732 (1992). 


\section{FIGURE CAPTIONS}

Fig. 1. Schematic of various physical processes during laser ablation, including plasma-solid interactions, laser-plume interactions, plasma expansion and transport, and plasmasubstrate interactions.

Fig. 2. The time evolution of the phase state of the finite difference cells for laser annealing of an amorphous $\mathrm{Si}$ layer on a crystal $\mathrm{Si}$ substrate.

Fig. 3. Schematic of electronic and photoelectric processes in the simple kinetic model for modeling of vapor breakdown.

Fig. 4. Output for particle densities as a function of time at energy fluence of $6.0 \mathrm{~J} / \mathrm{cm}^{2}$.

Fig. 5. A typical output from 1-D gas dynamic model for local fluid velocity (top panel), local sound velocity (middle panel), and local ionization fraction (bottom panel) as a function of time at energy fluence of $6.0 \mathrm{~J} / \mathrm{cm}^{2}$.

Fig. 6. Plasma expansion and transport with uniform initial velocity distribution in $x$ direction from 2-D particle hydrodynamic model.

Fig. 7. Density contours of particles in the plasma during free expansion into both vacuum and background gas from 2-D particle hydrodynamic model.

Fig. 8. 3-D isosurface plot of particle density (arbitrary units) in the expanding plasma with finite initial forward fluid velocity, indicating density peaks near the target surface from 2-D particle hydrodynamic model. 
Material processing by laser ablation

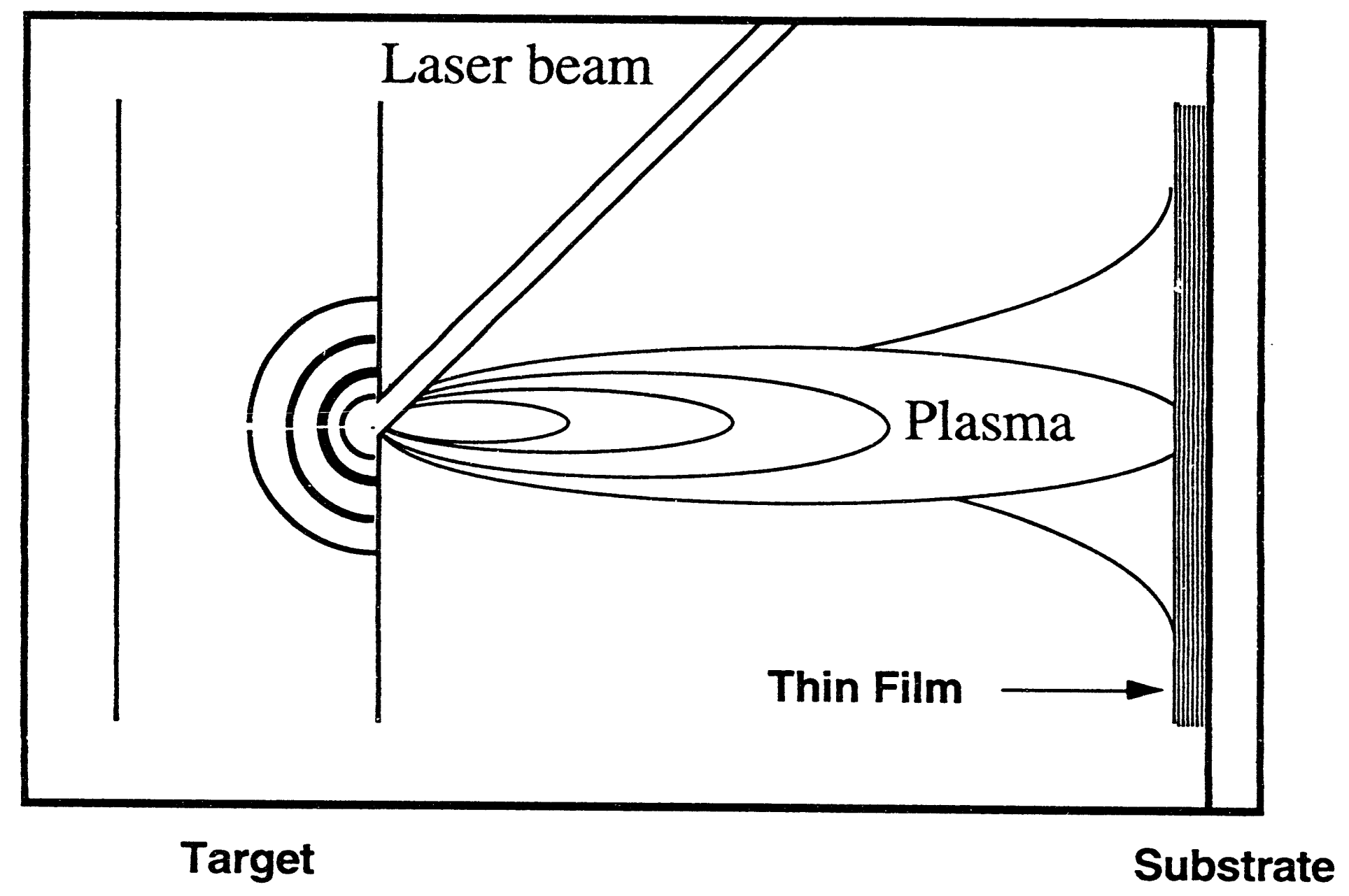

Fig. 1 
ORNL-DWG 86.8148A

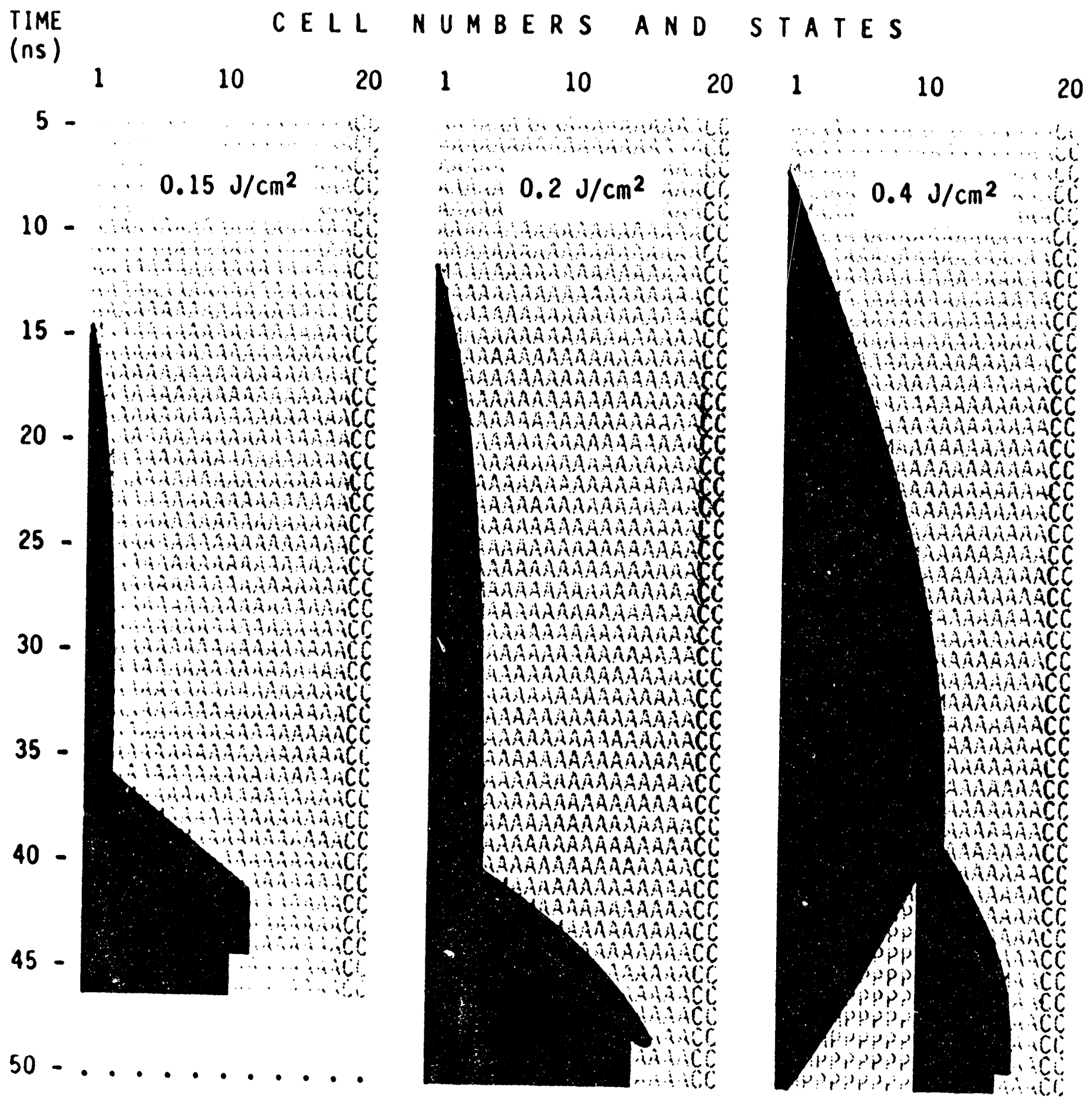

EXAMPLES OF LASER 8 OUTPUT

Fig. 2 


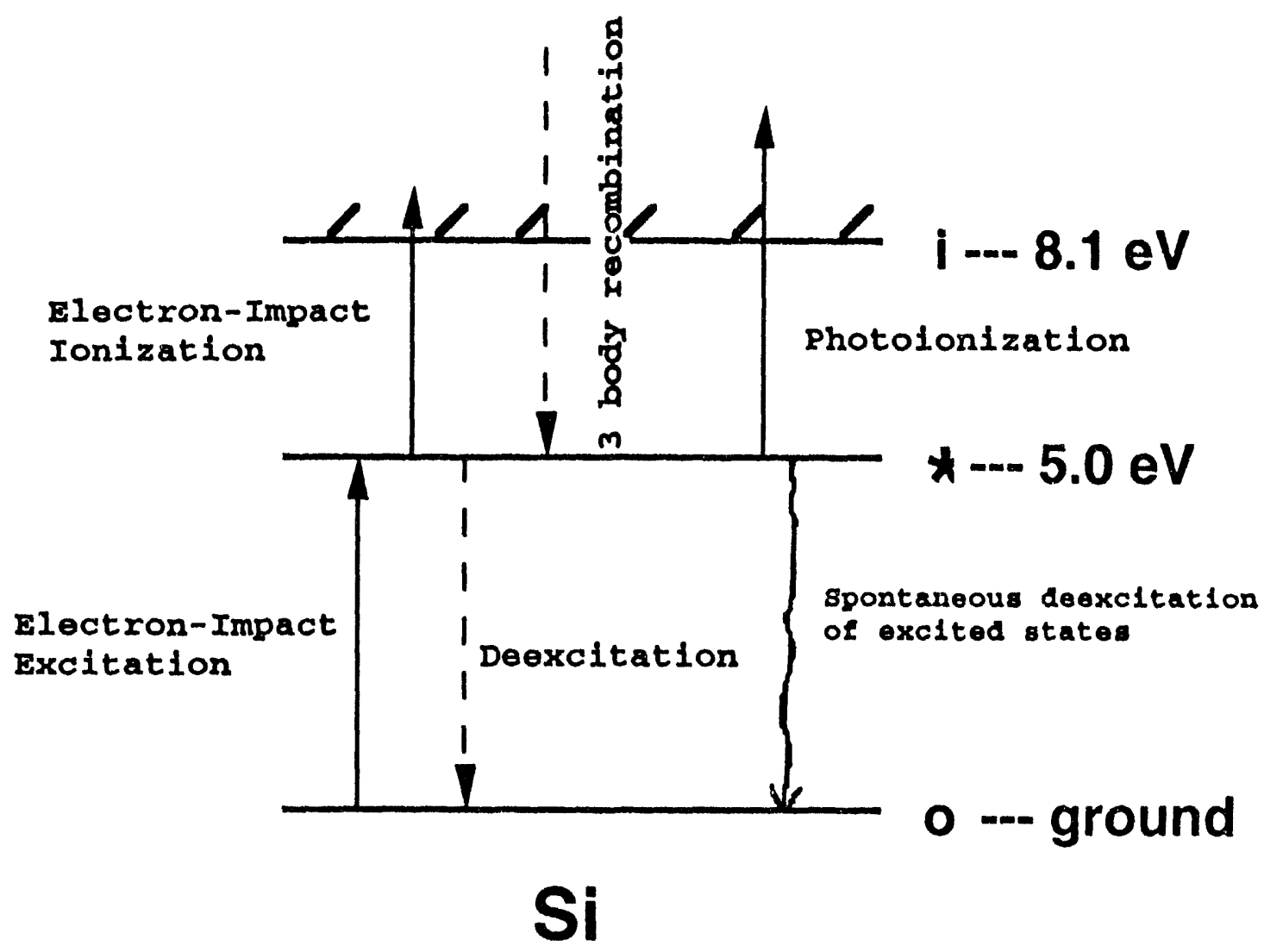

Fig. 3 
Particle density as a function of time in the plume (Energy fluence $=6.0 \mathrm{~J} / \mathrm{cm}^{2}$ )

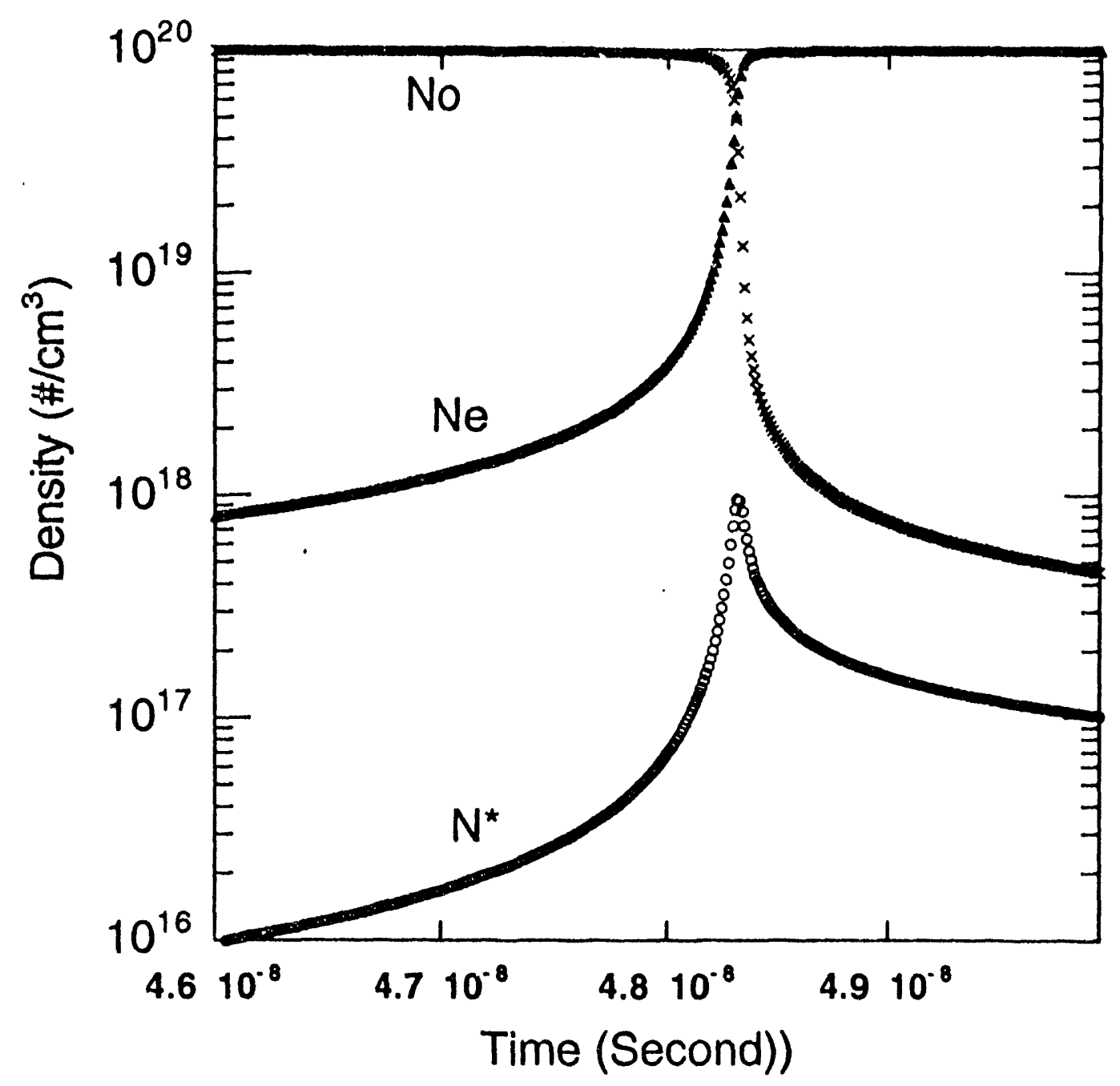

Fig. 4 

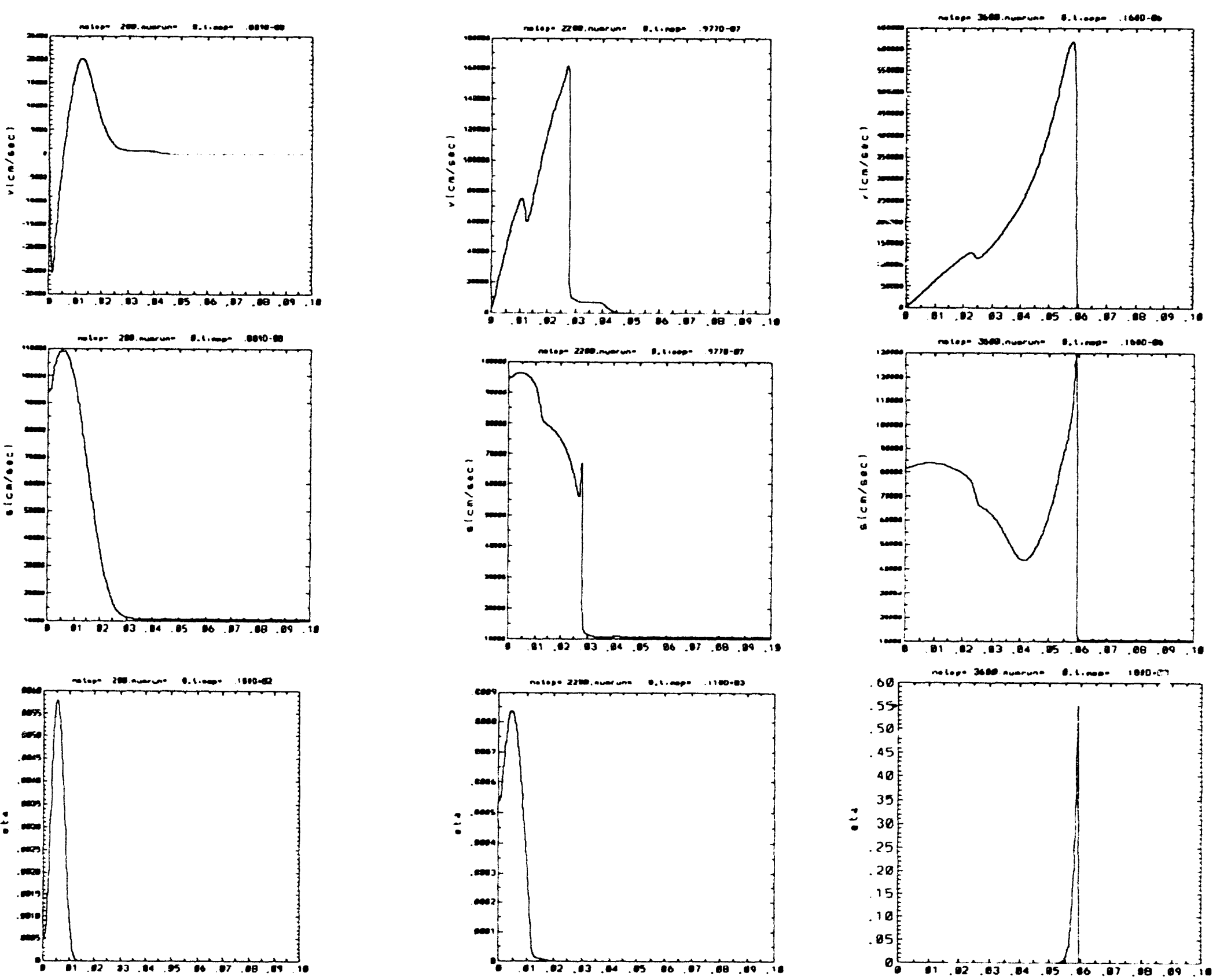

Fig. 5 

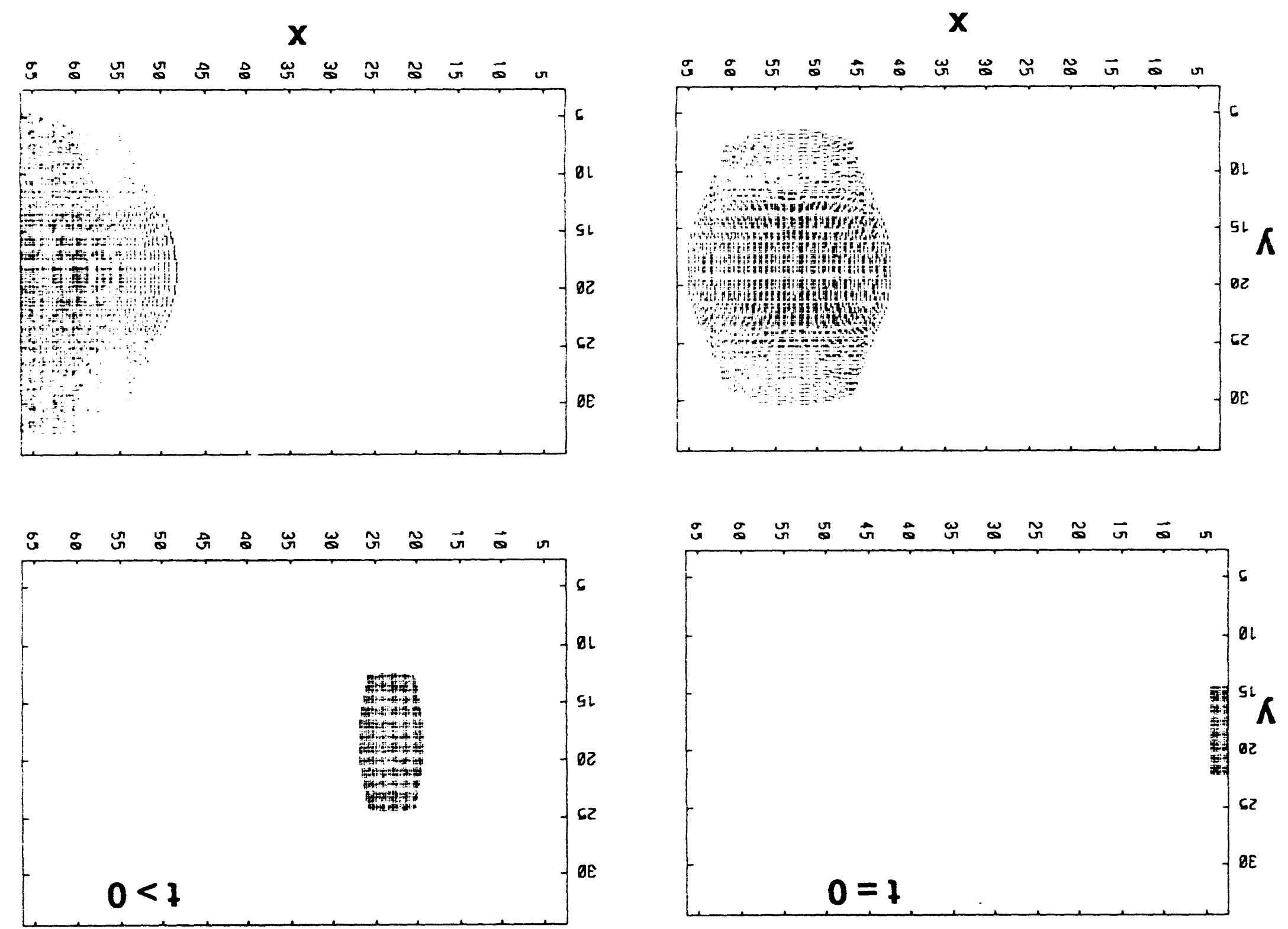

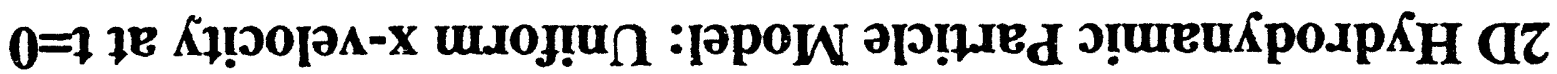
8uṛs 


\section{Free Expansion of Plume in Vacuum}
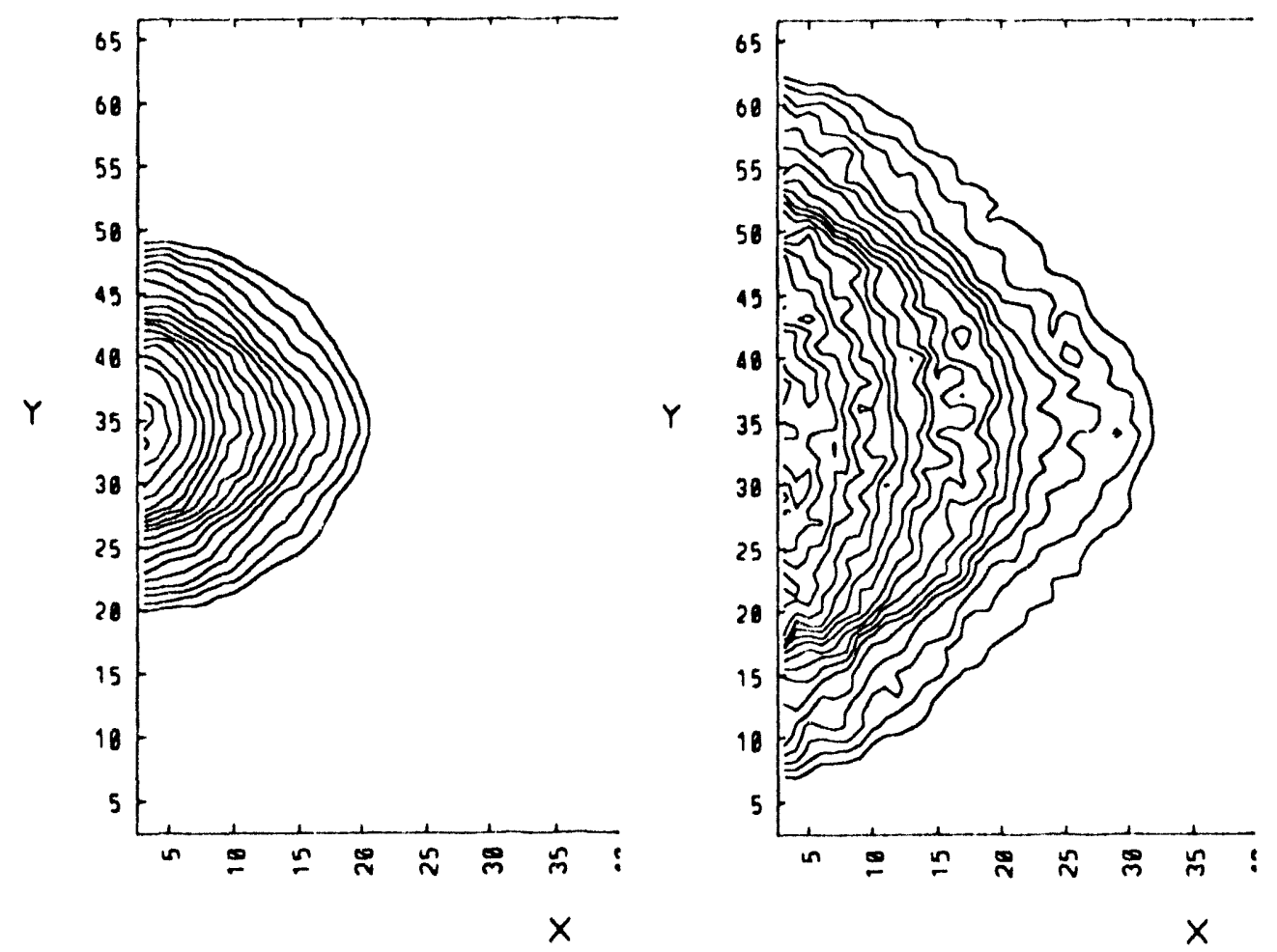

Free Expansion of Plume in Background Gas
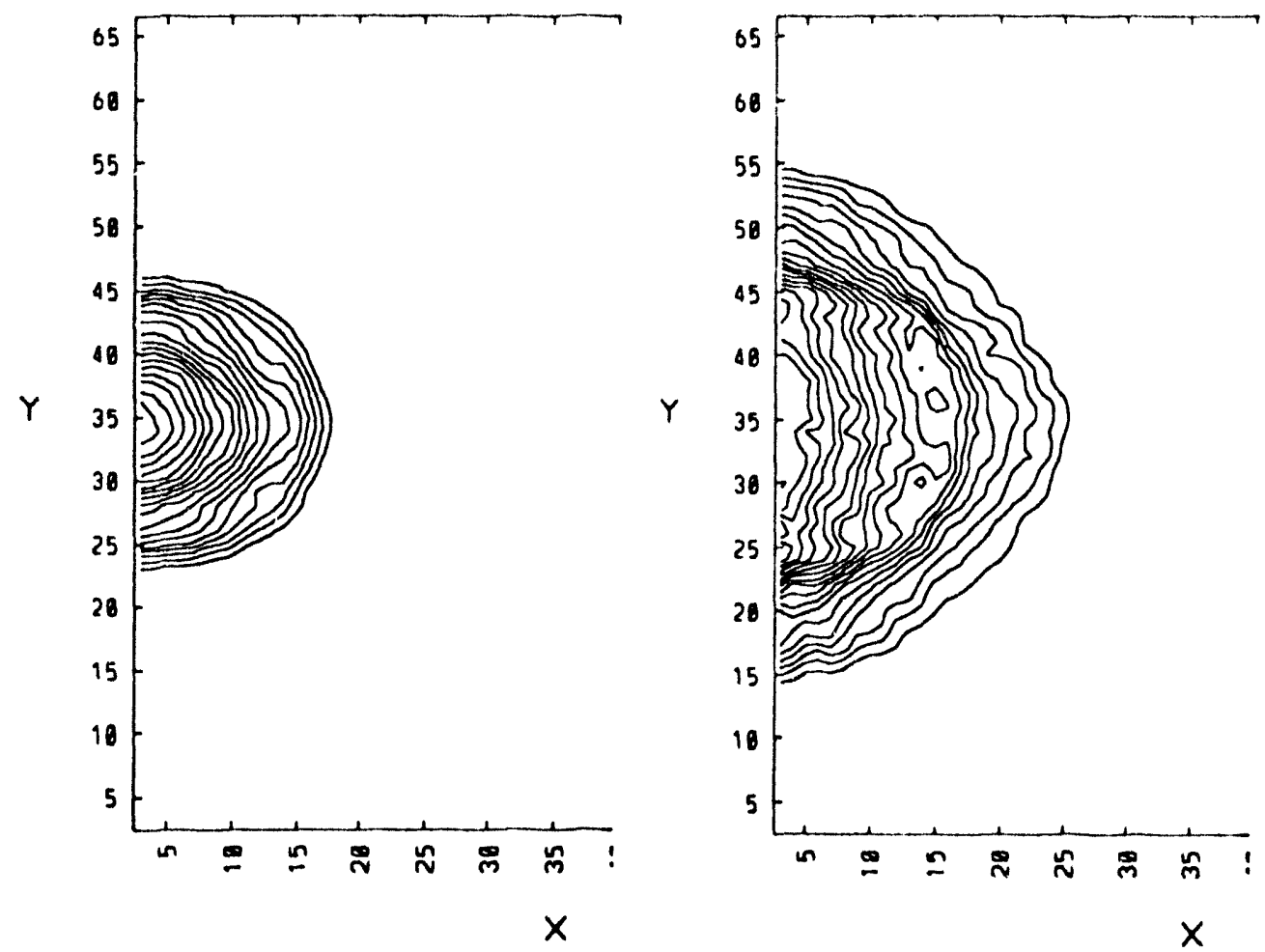

Fig. 7 


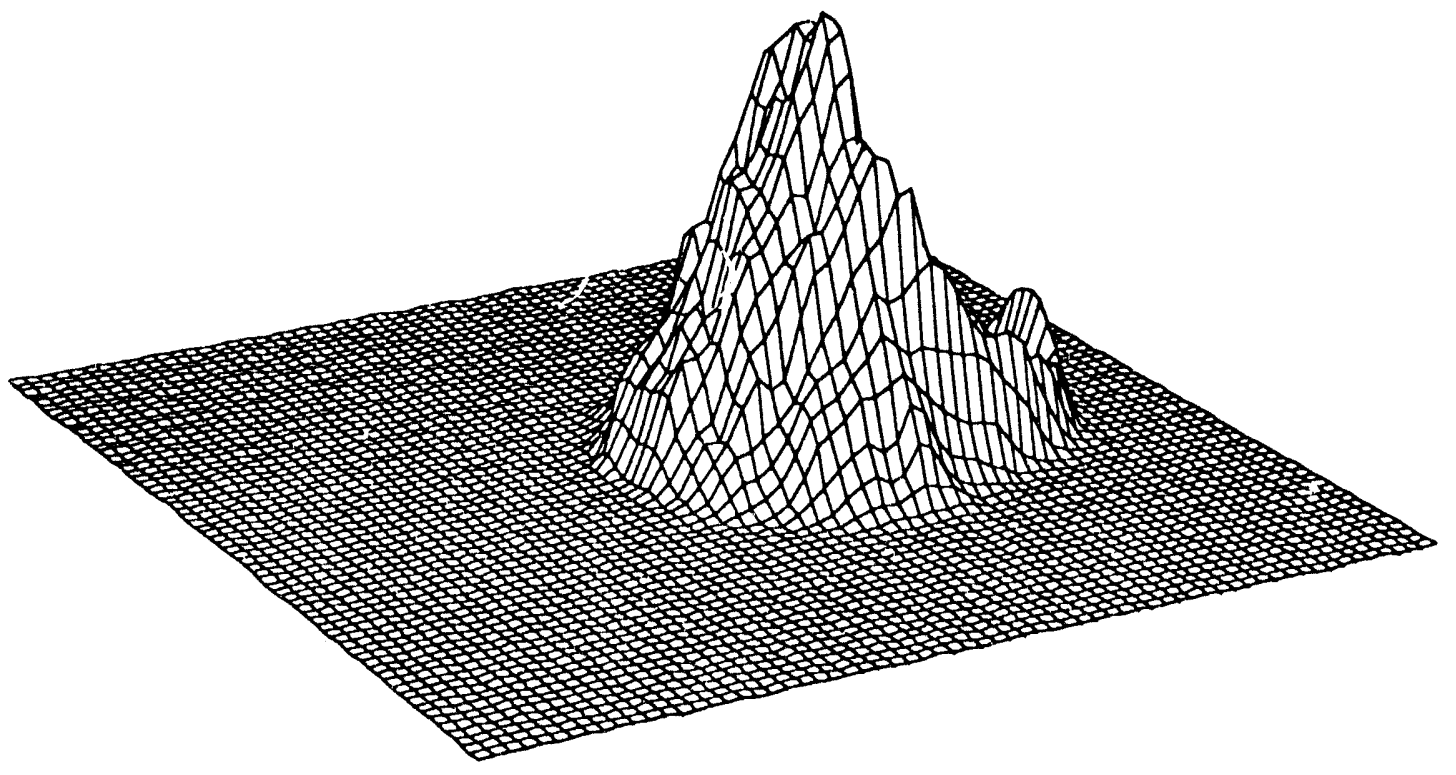

Fig. 8 

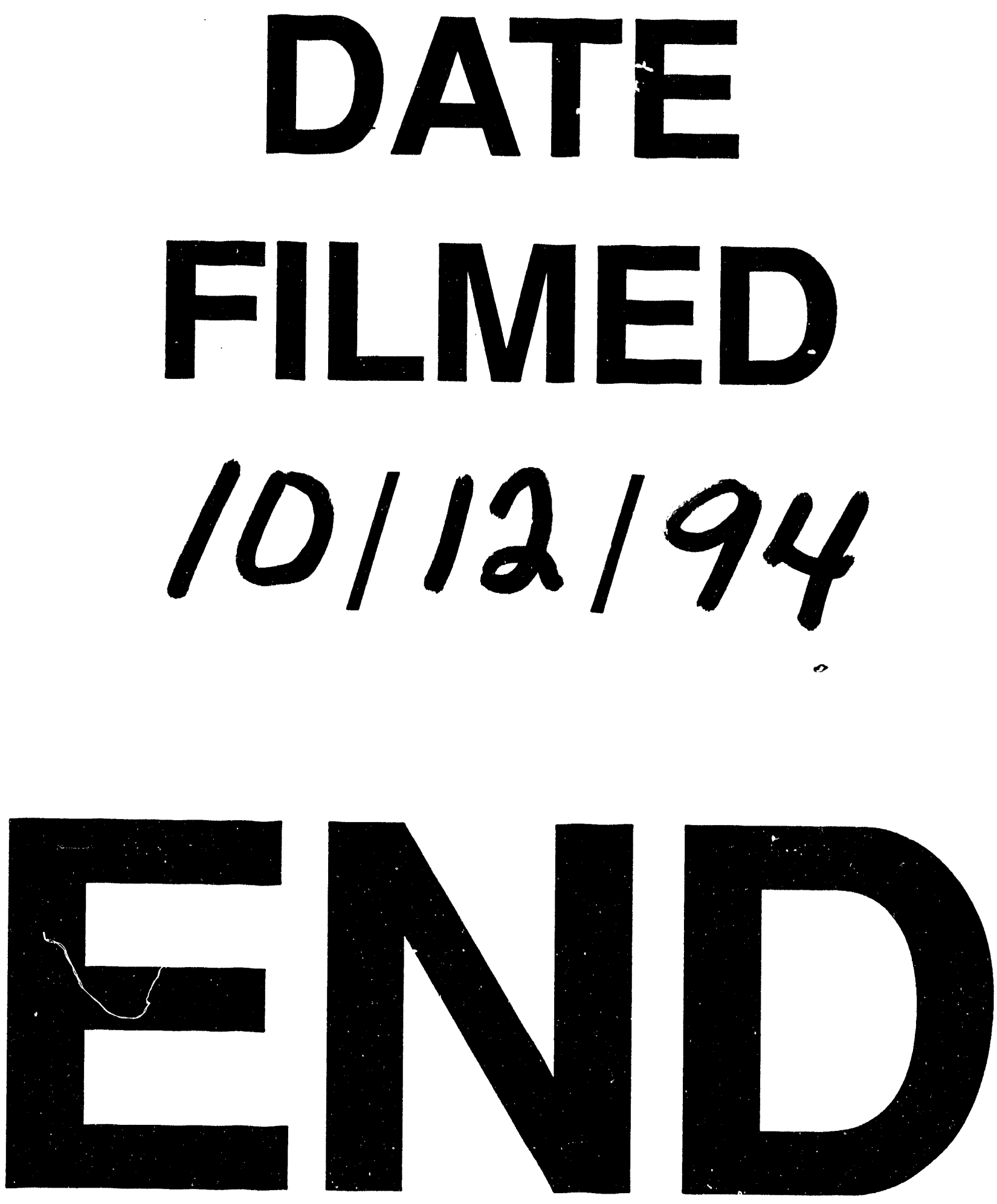
